\title{
Suicidal thoughts and behaviour among South African adolescents living with HIV: Can social support buffer the impact of stigma?
}

\author{
Marisa Casale, Mark Boyes, Marija Pantelic, Elona Toska and Lucie Cluver
}

\begin{abstract}
:
Background: Adolescents living with HIV represent a high-risk population for suicidal ideation and attempts, especially in low-income settings. Yet little is known about risk and protective factors for suicide in this population.

Methods: A moderated mediation model was employed to test for potential (a) effects of stigma on suicidal ideation and attempts, both direct and mediated through depression and (b) direct and stress-buffering effects of social support resources on depression and suicidal ideation and attempts, among 1053 HIV-positive 10-19-year-old adolescents from a resource-scarce health district in South Africa. The survey data was collected using full community sampling of 53 clinics and tracing to over 180 communities. Effects of two support resources were tested: perceived support availability from the adolescents' social network and structured clinic support groups. Stigma was measured using the ALHIV-SS scale, depression through the CDI short form and social support through items from the MOS-SS.

Results: Stigma was a risk factor for depression $(B=0.295 ; p<001)$ and for suicidal thoughts and behaviour $(B=0.185 ; p<.001)$. Only perceived support availability was directly associated with less depression $(B=-0.182, p<.001)$. However, both perceived support availability and support group participation contributed to the overall stress-buffering effects moderating the direct and indirect relationships between stigma and suicidal thoughts and behaviour.

Limitations: The data used in this study was self-reported and cross-sectional.

Conclusions: Findings suggest that strengthening multiple social support resources for HIVpositive adolescents, through early clinic and community-based interventions, may protect them from experiencing poor mental health and suicidal tendencies.
\end{abstract}

\section{Introduction}

Adolescents living with HIV (ALHIV) are a high-risk population for suicidal ideation and attempts. Worldwide, suicide accounts for an estimated $6 \%$ of all deaths among young people, representing the second leading cause of mortality among females and the third among males aged 10-24 years (McKinnon et al., 2016; Patton et al., 2009). Youth suicide is therefore a major global public health concern, especially in low and middle income countries (LMICs), which are home to around 90\% of the world's youth and account for 
over $75 \%$ of global suicide deaths (McKinnon et al., 2016; UNFPA, 2014). It is estimated that approximately $15 \%$ of adolescents aged 13-15 in LMICs experience suicidal ideation (i.e. are thinking of killing themselves) (Page et al., 2013), which in turn is strongly related to prospective suicidal attempts (McKinnon et al., 2016).

These risks are particularly salient in countries and populations with high HIV prevalence, given elevated rates of suicidal ideation and attempts found among people living with HIV (Catalan et al., 2011). A meta-analysis of data from 32 LMICs found rates of suicidal ideation to be highest among adolescents in the African region, and HIV/AIDS was posited to be a contributing factor (McKinnon et al., 2016). However very little is known about suicidal thoughts and behaviours among ALHIV in Africa, including potential risk and protective factors (McKinnon et al., 2016). Moreover, evidence of suicide management and prevention programs for this population is scant (Catalan et al., 2011).

HIV-related stigma is a potential risk factor for poor mental health and suicide among ALHIV. Decades into the HIV epidemic and despite numerous reduction efforts, stigma persists in many HIV-affected populations, and continues to act as barrier to HIV treatment and care (Dow et al., 2016; Katz et al., 2013; Rueda et al., 2016). ALHIV in particular may experience exceptionally high levels of stigma and discrimination (HIV Leadership through Accountability Programme: GNP and NAPWA SA, 2012). Recent metaanalyses point to higher rates of depression, anxiety and emotional distress among people living with HIV who perceive more HIV-related stigma (Katz et al., 2013; Rueda et al., 2016); reviewed studies include longitudinal studies and randomised control trials. More specifically, HIV-related stigma has been associated with worse mental health among HIVpositive and HIV-affected youth, also in longitudinal analyses (Boyes and Cluver 2013; Cluver and Orkin, 2009; Dow et al., 2016; Pantelic et al., 2017), and with suicidal ideation and attempts among adults living with HIV (Bitew et al., 2016; Gebremariam et al., 2017). Links between poor mental health and suicide are well established, including among adolescents (Wild et al., 2004). Adolescence is a high risk period for the development of mental health problems (Kessler et al., 2012) and there is evidence to suggest that ALHIV may be at elevated risk of mental health disorders (Scharko, 2006; Mellins and Malee, 2013; Willis et al., 2018). Moreover, several Southern African studies, including longitudinal analyses, have shown poor mental health to mediate the relationship between specific stressors, such as parental death and abuse, and suicidal thoughts and behaviours among adolescents (Cluver et al., 2015; Shilubane et al., 2014). However none of these studies have examined the potentially interruptible pathways between HIV-related stigma and suicidal thoughts and behaviour with ALHIV.

In contrast, social support may be an important protective mechanism for adolescent psychosocial health. Social support in relation to health has been described as supportive relationships that provide something that people need to stay healthy or adapt to stress (House et al., 1988). These relationships may provide various types of support, including emotional, informational and instrumental assistance: this functional content of social relationships has been more strongly and frequently associated with better mental health 
outcomes than structural dimensions of support (e.g. quantity and type of inter-personal relationships) (Cohen and Wills, 1985; Ryan and Willits, 2007). The most important 'sources' or providers of this support in an individual's informal support network are most commonly friends, family and significant others (Thoits, 2011), but sources may include other providers such as church and other community groups. Moreover, perceived social support - i.e. the perception of the availability and adequacy of support from one's network of family, friends and others - has been more strongly and consistently linked to mental health outcomes than retrospective measures of actual support received (McDowell and Serovich, 2007; Thoits, 2011). This may be explained by the fact that perceived support is less likely to be confounded with the severity of stress and support needs over a particular time period, and instead more likely to derive from generalisations based on multiple and regular experiences of support (Thoits, 2011).

A large body of research links social support to better mental health and lower odds of suicide among people living with HIV or other chronic illness (Bitew et al., 2016; Rzeszutek, 2017; Umberson and Montez, 2010). Higher social support (especially from caregivers) has been found to be protective against mental health difficulties among ALHIV (Gentz et al., 2017) and for suicidal behaviours among youth in high-income countries; this includes evidence from longitudinal studies (McKinnon et al., 2016; Teismann et al., 2016). Social support can impact these psychological outcomes either by means of a direct effect, or by buffering the negative effects of specific stressors on these outcomes (Alloway and Bebbington, 1987; Casale et al., 2015a; Gellert et al., 2018). While multiple studies show direct protective effects of social support on depression and suicide among people living with HIV (Bitew et al., 2016; Brandt, 2009; Gentz et al., 2017), the evidence for stress-buffering is mixed: some studies have found social support to moderate effects of HIV-related stressors, such as financial stress or HIV-illness, on mental health outcomes (Robbins et al., 2003; Wight et al., 2003), while others did not find stressbuffering effects for HIV-related stigma (Breet et al., 2014; Rueda et al., 2012). However none of these studies were conducted specifically with adolescent populations in LMICs.

It should also be noted that most empirical evidence on protective effects of social support for health and mortality has been based on "naturally occurring social relationships" (Holt-Lanstad et al., 2010). There have been many attempts to replicate or complement these positive effects through interventions, some of which have shown promise, and others that have proved less effective or simply not financially feasible in certain contexts (Ertel et al., 2010; Feaster et al., 2010; Holt-Lanstad et al., 2010). Prompted by broader evidence of the potential positive effects of social support on a range of health outcomes and behaviours (Holt-Lanstad et al., 2010; Umberson and Montez, 2010), HIV support groups have been established across sub-Saharan Africa by governments, health facilities and community-based organizations, to provide socio-emotional support to people living with HIV while they initiate and maintain treatment. While evidence on the effectiveness of these interventions is limited, recent cross-sectional and longitudinal research suggests that these HIV support groups - run by nurses or lay counsellors in government facilities providing treatment for patients living with HIV - may have a positive impact on adherence 
to medication and overall health of adults and adolescents living with HIV (Cluver et al., 2016; Wouters et al., 2009). Support group interventions have also been found to improve mental health and psycho-social functioning among youth living in families affected by HIV (Smith Fawzi et al., 2012; Wu and Li, 2013). But there is no evidence of whether and how HIV support groups may affect the mental health of ALHIV.

This paper investigates potential direct and stress-buffering effects of social support resources on depression and suicidal ideation and attempts, among ALHIV in resourcescarce South African communities. Specifically, this study investigates the potential protective effects of two dimensions of social support against depression and suicidal tendencies: perceived availability of social support (commonly provided through 'naturally occurring' social networks and relationships (Holt-Lanstad et al., 2010)), and support groups linked to clinics (an example of a support 'intervention' provided by public health facilities).

We hypothesised that: (i) stigma would be associated with higher suicidal thoughts and behaviour, directly and/or mediated by increased depression; (ii) support resources, specifically perceived social support availability and HIV support group participation, would be directly associated with less depression and suicidal thoughts and behaviour; and (iii) that these support resources would buffer the effects of stigma on depression and suicidal thoughts and behaviour.

\section{Methods}

\subsection{Sampling and data collection}

Data were collected in 2014-2015 from 1053 adolescents 10-19 initiated on HIV treatment within a high HIV-prevalence resource-limited health district in South Africa's Eastern Cape province. The study was developed in collaboration with the South African Departments of Health and Basic Education, UNICEF, PEPFAR-USAID, and regional and local NGOS. All public healthcare facilities providing HIV treatment to five or more ALHIV in the district were selected for inclusion $(n=53)$. ALHIV who had initiated HIV treatment were identified through clinic patient records $(n=1176)$ and traced back to their communities $(n=180)$, to ensure inclusion of those not engaged in care. Adolescents were recruited and interviewed at the clinics or in their communities (in their homes, schools or other locations they identified as safe). The large majority $(n=1053 ; 90 \%)$ of eligible adolescents were interviewed; $4 \%$ of adolescents or their caregivers refused participation; around $1 \%$ were unable to participate because of severe cognitive delay, and the remaining $5 \%$ were not traceable. The pre-piloted questionnaire was developed in English, and items were translated and back translated independently. One-on-one tablet-assisted 60-90 min interviews were conducted with the adolescents in the language of their choice (for the large majority in Xhosa), by researchers trained in working with vulnerable adolescents. Data were linked to questionnaire serial numbers instead of participant names, to ensure anonymity. Incentives were not provided, but participants were given certificates, refreshments, and a toiletry pack, regardless of whether they completed the interview. Voluntary informed consent was obtained from caregivers and adolescents. For cases of 
reported recent abuse, rape, suicidal attempt or other risk of significant harm, referrals were made to child protection and health services $(n=66,6.2 \%)$.

Ethical clearance for this study was provided by Oxford University (SSD/CUREC2/12-21), the University of Cape Town (CSSR 2013/4), the South African National Departments of Health, Basic Education and Social Development, and the Eastern Cape Departments of Health, Basic Education and Social Development.

\section{Measures}

\subsection{Outcome variable: suicidal thoughts and behaviour}

Suicidal thoughts and behaviour were measured using the Mini International Psychiatric Interview for Children and Adolescents Suicidality and Self-harm subscale (Sheehan et al., 2004). The scale contains four questions on suicidal ideation (i.e. whether the adolescent had wished he/she were dead or thought about killing or hurting him/ herself in the previous month) and one on suicidal attempt (i.e. whether the adolescent had tried to kill him/herself in the previous month); response options are binary (1: yes; o: no). The MINI-Kid has been extensively validated, and shows strong internal consistency and test-retest reliability (Lecrubier et al., 1997). For this sample $\alpha=0.88$.

\subsection{Independent variable: stigma}

Stigma was measured using the 10-item ALHIV stigma scale (ALHIV-SS); this consists of items measuring internalized stigma ( 5 items), anticipated stigma ( 2 items) and enacted HIV stigma (3 items). Response options are: 0: never; 1: sometimes; 2: most of the time. The scale has been cross-culturally adapted from a stigma scale previously used with HIVpositive adolescents in the US (Wright et al., 2007), through cognitive interviews with South African adolescents, and validated within the present study (Pantelic et al., 2018). $\alpha$ $=0.66$ in this sample.

\subsection{Mediator: depression}

Severity (o: never; 1: many times; 2: all the time) of depressive symptoms was measured using the Children's Depression Inventory (CDI 10-item short form) (Kovacs, 1992). The CDI is a widely used and well-validated measure with strong psychometric properties. It has been used with various other South African populations, including AIDS-affected children (Cluver et al., 2015; Suliman, 2002). $\alpha=0.64$ in this sample.

\subsection{Moderators: perceived social support availability and HIV support group participation}

Perceived social support availability was measured using seven items from the Medical Outcome Study Social Support Survey/MOS-SS (Sherbourne and Stewart, 1991), an instrument that measures functional support. Three items measured instrumental support (e.g. support with preparing meals or being accompanied to a doctor) and four items measured emotional/informational support (e.g. advice or assistance in dealing with personal problems). This scale has been widely used in the developing world, including with HIV-affected populations in South Africa (Casale et al., 2015b). It demonstrated good 
reliability of $\alpha=0.85$ for this sample. For support group participation, adolescents were asked how often they attended an HIV support group at their clinic (weekly, monthly, yearly, once in a while, no attendance). Given the low frequency of any support group attendance overall (18\%) this variable was dichotomized to: 1 : attendance; $o$ : no attendance.

\subsection{Socio-demographic and HIV control variables}

Covariates included in these analyses are: age, gender, urban versus rural location, socioeconomic status, whether the child's primary caregiver is a biological parent, and mode of HIV infection. Relative socio-economic status (SES) was measured through a composite scale comprising the top eight socially-perceived necessities for children $(\alpha=0.72)$ as identified by the Centre for South African Social Policy (Wright, 2008), and endorsed by over $80 \%$ of the South African population in the nationally-representative South African Social Attitudes Survey 2006 (Pillay et al., 2007); these include items such as 'enough clothes to keep you warm and dry' and ' 3 meals a day'. The existence of a primary caregiver and his/her relationship to the adolescent was identified using items from the National Survey of HIV and Risk Behaviour amongst young South Africans (RHRU, 2005). Mode of HIV infection was included as an additional control variable given recent evidence of worse mental health among youth with behaviourally acquired HIV (Lewis et al., 2015). It was assessed based on cohorts of children and adolescents living with HIV in Southern Africa (Slogrove et al., 2017). Vertical infection refers to acquiring HIV through birth or breastfeeding and horizontal infection refers to acquiring HIV later on in life, mainly through sexual intercourse in this context. Adolescents were coded as vertically-infected if they had started treatment before age 10, with allocations verified using a predictive algorithm of literature-informed factors (e.g. orphanhood as a predictor of vertical infection and sexual abuse as a predictor of horizontal infection).

\subsection{Data analyses}

The analyses were conducted in three stages. First, partial correlations were run in SPSS to identify significant associations between all key variables in the hypotheses to be tested, after controlling for socio-demographic covariates: stigma, perceived social support availability, support group participation, depression and suicidal thoughts and behaviour. The second step consisted of running the moderated mediation model, using the PROCESS plug-in for SPSS (Model 76, Hayes, 2013). This analysis was used to test all three hypotheses indicated above, i.e. for: direct effects of stigma on suicidal thoughts and behaviour and indirect effects mediated by depressive symptoms; direct associations between the support resources and depression and suicidal thoughts and behaviour; and moderation effects of the two support variables on the direct and indirect relationships between stigma and suicidal thoughts and behaviour. All continuous variables were standardized and the analysis controlled for socio-demographic covariates. Boot-strapping, a non-parametric sampling procedure, was used to simultaneously test for direct and indirect effects of the mediator (Preacher and Hayes, 2008); for 1000 bootstrap samples, results are statistically significant where $95 \%$ confidence intervals do not overlap zero. The third and final step consisted of illustrating and probing significant interactions using simple slopes tests, in order to further test the moderation effects (Aiken and West, 1991; Dawson, 2018). 


\section{Table 1}

Descriptive statistics and frequencies $(n=1053)$.

$\begin{array}{ll}\text { Socio-demographic covariates } & \\ \text { Male }(n, \%) & 476(45) \\ \text { Urban location }(n, \%) & 823(78) \\ \text { Horizontally acquired HIV }(n, \%) & 267(25) \\ \text { Caregiver is biological parent }(n, \%) & 474(45) \\ \quad \text { Age (mean, SD) } & 13.82(2.84) \\ \text { Socio-economic status (mean, SD) } & 1.61(1.80) \\ \text { Main } I V & \\ \quad \text { Stigma (mean, SD) } & 1.14(1.75) \\ \text { Mediator } & 1.26(1.98) \\ \quad \text { Depression (mean, SD) } & \\ \text { Moderators } & 12.92(2.17) \\ \quad \text { Perceived social support (mean, SD) } & 188(18) \\ \text { Support group attendance }(n, \%) & \\ \text { Outcome variable } & 1.19(0.78) \\ \quad \text { Suicidal thoughts and behaviour (mean, SD) }\end{array}$

\section{Results}

\subsection{Descriptive statistics}

Sample characteristics and descriptive statistics for the variables of interest are summarized in Table 1 . The average age of included adolescents was 14 and $78 \%$ were living in urban areas. The large majority (75\%) had acquired HIV through vertical infection. 43.5\% reported having experienced some level of stigma and just under half of the sample (46\%) were experiencing one or more symptom(s) of depression. Eight percent (84 adolescents) reported some level of suicidal thoughts over the previous month and approximately half (4\%) of these participants indicated that they had tried to kill themselves during that period. Around $18 \%$ of participants attended a support group at their clinic.

\subsection{Partial correlations}

Table 2 shows partial correlations for key variables for the analyses, after controlling for covariates. Partial correlations between stigma, depression, perceived social support and suicidal thoughts and behaviour are in the expected direction and small to moderate in size ( $p<.001$ for all associations). Support group attendance, instead, is not significantly correlated with any of the other key variables. In particular, the lack of a significant association between the MOS social support scale and participation in a support group, and the small effect size of the association $(B=-0.027)$, indicate that these variables are distinct constructs likely measuring different functional dimensions of support. 


\subsection{Moderated mediation analysis}

4.3.1 Direct and indirect effects of stigma on suicidal thoughts and behaviour

Tables $3-5$ and Fig. 1 display the results of the moderated mediation analysis. Higher HIVrelated stigma is directly associated with higher depressive symptoms $(B=0.295 ; p<$ $.001)$ and higher suicidal thoughts and behaviour $(B=0.185 ; p<.001)$ in this sample.

Table 2

Partial correlations between stigma, support resources, depression and suicidal thoughts and behaviour.

\begin{tabular}{lllll}
\hline & Stigma & $\begin{array}{l}\text { Perceived } \\
\text { social support }\end{array}$ & Depression & $\begin{array}{l}\text { Suicidal } \\
\text { thoughts and } \\
\text { behaviour }\end{array}$ \\
\hline $\begin{array}{l}\text { Stigma } \\
\text { Perceived social } \\
\text { support }\end{array}$ & $-0.185^{* *}$ & - & & \\
$\begin{array}{c}\text { Depression } \\
\text { Suicidal thoughts } \\
\text { and behaviour }\end{array}$ & $0.308^{\cdots *}$ & $-0.259^{\cdots *}$ & - & \\
$\begin{array}{c}\text { Support group } \\
\text { attendance }\end{array}$ & 0.022 & -0.027 & -0.036 & -0.020 \\
\hline
\end{tabular}

Note: all correlations controlled for age, gender, location, socio-economic status, mode of infection and whether caregiver is biological parent.

${ }^{* *} p<.01$.

${ }^{*} p<.05$.

${ }^{* * *} p<.001$. 
Table 3

Results of PROCESS Model 76 multivariate regression predicting depression.

\begin{tabular}{llll}
\hline & $\begin{array}{l}B \text { (unstandardized } \\
\text { coefficient) }\end{array}$ & LLCI & ULCI \\
& & & \\
\hline Socio-demographic covariates & 0.068 & -0.004 & 0.139 \\
$\quad$ Age & -0.002 & -0.115 & 0.112 \\
$\quad$ Male & 0.090 & -0.048 & 0.228 \\
$\quad$ Urban location & $0.063^{*}$ & 0.006 & 0.120 \\
$\quad$ Worse SES & 0.096 & -0.065 & 0.257 \\
$\quad$ Horizontal infection & -0.071 & -0.185 & 0.043 \\
$\quad$ Caregiver biological parent & & & \\
Stressor/IV & $0.295^{* *}$ & 0.228 & 0.361 \\
$\quad$ Stigma & $-0.182^{* * *}$ & -0.242 & -0.121 \\
Moderators & -0.113 & -0.258 & 0.033 \\
$\quad \begin{array}{l}\text { Perceived social support } \\
\text { Support group attendance }\end{array}$ & $-0.077^{* *}$ & -0.121 & -0.034 \\
Interactions & & -0.370 & 0.090 \\
$\quad$ Stigma $\times$ perceived social & support & & \\
$\quad$ Stigma $\times$ support group & $-0.230^{* *}$ & & \\
\hline
\end{tabular}

Model summary: $R=0.453 ; R-s q=0.206 ; F=23.244$.

${ }^{* * *} p<.001$.

${ }^{* *} p<.01$.

${ }^{*} p<.05$.

Table 4

Results of PROCESS Model 76 multivariate regression predicting suicidal thoughts and behaviour.

\begin{tabular}{|c|c|c|c|}
\hline & $\begin{array}{l}B \text { (unstandardized } \\
\text { coefficient) }\end{array}$ & LLCI & ULCI \\
\hline \multicolumn{4}{|l|}{ Socio-demographic covariates } \\
\hline Age & 0.031 & -0.024 & 0.085 \\
\hline Male & -0.068 & -0.154 & 0.018 \\
\hline Urban location & -0.031 & -0.136 & 0.074 \\
\hline Worse SES & -0.002 & -0.045 & 0.042 \\
\hline Horizontal infection & 0.066 & -0.056 & 0.189 \\
\hline Caregiver biological parent & -0.054 & -0.141 & 0.032 \\
\hline \multicolumn{4}{|l|}{ Stressor/IV } \\
\hline Stigma & $0.185^{* *}$ & 0.132 & 0.238 \\
\hline \multicolumn{4}{|l|}{ Mediator } \\
\hline Depression & $0.164^{\cdots *}$ & 0.109 & 0.220 \\
\hline \multicolumn{4}{|l|}{ Moderators } \\
\hline Perceived social support & 0.025 & -0.022 & 0.072 \\
\hline Support group attendance & -0.017 & -0.127 & 0.094 \\
\hline \multicolumn{4}{|l|}{ Interactions } \\
\hline $\begin{array}{l}\text { Stigma } \times \text { Perceived social } \\
\text { support }\end{array}$ & -0.002 & -0.040 & 0.036 \\
\hline Stigma $\times$ Support group & $-0.112^{*}$ & -0.222 & -0.002 \\
\hline $\begin{array}{l}\text { Depression } \times \text { Perceived } \\
\text { social support }\end{array}$ & $-0.074^{* *}$ & -0.106 & -0.042 \\
\hline Depression $\times$ Support group & -0.000 & -0.119 & 0.119 \\
\hline
\end{tabular}

Model summary: $R=0.476 ; R$-sq $=0.226 ; F=20.555$.

${ }^{\star *} p<.01$.

*** $p<.001$.

${ }^{*} p<.05$. 


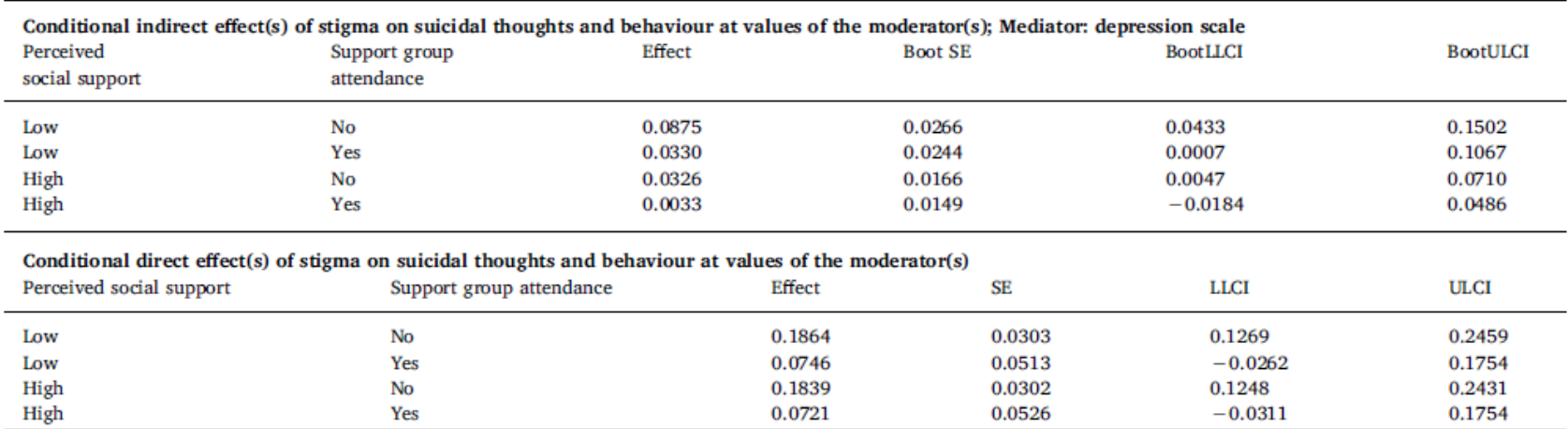

Depression is also associated with higher suicidal thoughts and behaviour scores $(B=$ 0.164; $p<.001)$. There are both significant direct effects of stigma on suicidal thoughts and behaviour and indirect effects via depression, dependent on the values of the moderator variables. This indicates the presence of moderated mediation, as discussed below and in Table 5 .

\subsubsection{Direct associations between the support variables, depression and suicidal thoughts and behaviour}

Regression results also show that higher perceived social support is directly and significantly associated with less depression $(B=-0.182 ; p<.001)$, while being part of a support group is not. Neither of the two support resources is directly associated with suicidal thoughts and behaviour.

\subsubsection{Moderating effects of perceived social support and HIV support group attendance}

Conditional direct and indirect effects of stigma on suicidal thoughts and behaviour, at high and low levels of the respective moderators, are presented in Table 5. At low levels of perceived social support, there is a significant indirect relationship between stigma and suicidal thoughts and behaviour - mediated by depression - irrespective of whether or not adolescents participate in a support group (confidence intervals do not cross zero). This relationship remains significant for adolescents with high social support, who are not participating in a support group. However, in the presence of both support resources - high perceived social support and support group participation - the association is not significant (CIs [95\%]: -0.018-0.049). The presence of moderation is reinforced by the significant interactions in the regression analyses: both the stigma $\times$ social support scale $(B=$ $-0.077 ; p<.001)$ and stigma $\times$ support group $(-0.23 ; p<.01)$ interactions are significant predictors of depression, and the depression $\times$ support group interaction is significant for suicidal thoughts and behaviour $(B=-0.074 ; p<.001)$ (see Fig. 1). Instead, with regard to conditional direct effects, the direct relationship between stigma and suicidal thoughts and behaviour is significant only for adolescents who are not part of a support group, regardless of whether their perceived level of social support availability is high or low $(p<.001$ in both these cases). For adolescents who are part of a support 
group, this direct relationship is not significant. Evidence of support group moderation is reinforced by the significance of the stigma $\times$ support group interaction in predicting suicidal thoughts and behaviour $(B=-0.112 ; p<.05)$. The stigma $\times$ social support scale interaction is not significant.

\subsubsection{Interaction plots and slope tests}

The plots and slope tests for each of the four significant interaction terms, included in Supplementary Figs. 1-4, reinforce evidence of moderation effects. Slope gradients are significant for all significant interactions tested, and gradients are higher at lower levels of support resources (i.e. at lower levels of perceived social support or in the absence of support group participation).

\section{Discussion}

\subsection{Prevalence of suicidal thoughts and behaviour}

Our data indicate that 1 in approximately 12 ALHIV had thought about killing him/herself in the preceding month, and about half of these adolescents had attempted to do so. These rates are in line with findings of previous Southern African studies conducted with HIVaffected adolescents or HIV-positive adults attending clinics (7-8\% for suicidal ideation and just over 3\% for suicidal attempts (Cluver et al., 2015; Rukundo et al., 2016)). They are, however, lower than aggregate rates reported for people living with HIV globally or youth in South Africa and other LMICs (between 15 and 27\%) (Catalan et al., 2011; Page et al., 2013; The South African Depression and Anxiety Group, 2016) and are likely to be considerably higher if past-year or any life-time experiences are taken into consideration.
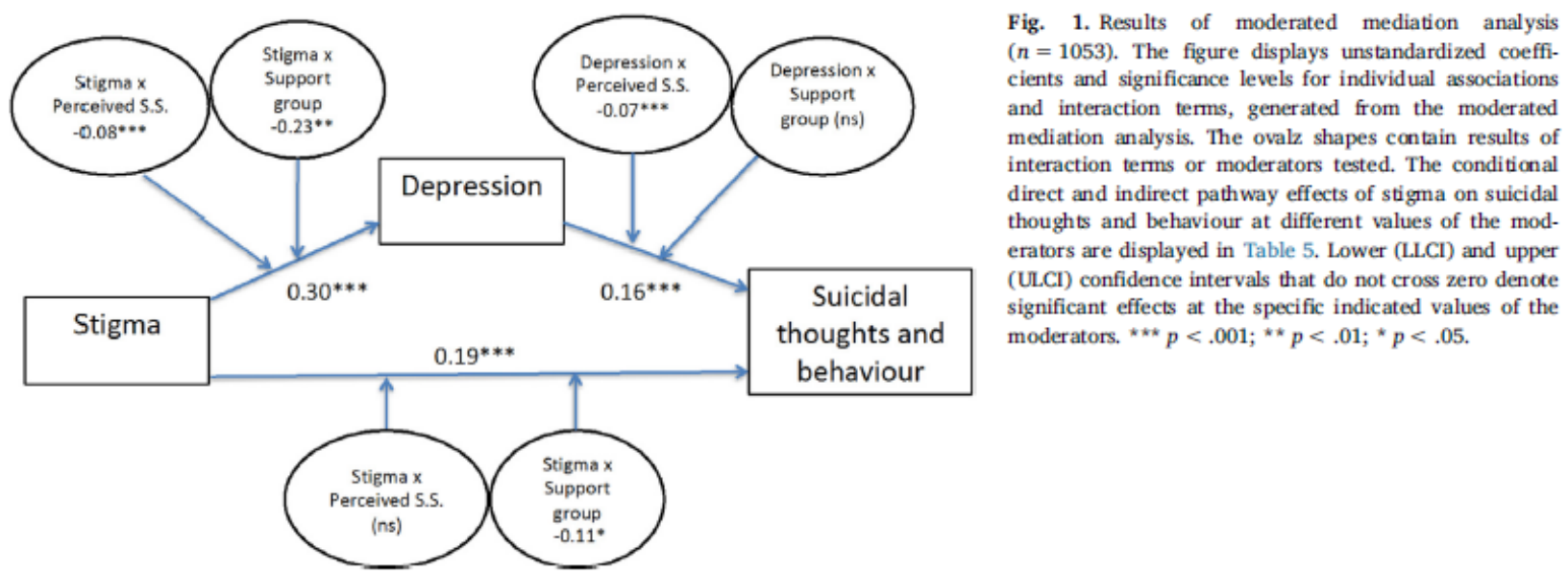

\subsection{Associations between stigma and suicidal thoughts and behaviour}

We hypothesised that stigma would be associated with higher suicidal thoughts and behaviour in this population of ALHIV, directly and/ or mediated by increased depression. Almost half of the adolescents in this sample (44\%) have experienced or perceived some level of HIV-related stigma, evidence of how pervasive this phenomenon still is. This study reinforces stigma as a risk factor for psychological distress and suicide among ALHIV: it identified both direct associations with suicide, in line with previous studies conducted with 
people living with HIV (Bitew et al., 2016; Gebremariam et al., 2017), as well as indirect effects mediated by depression.

\subsection{Direct associations between support resources, depression and suicidal thoughts and behaviour}

We hypothesised that support resources, specifically perceived social support availability and HIV support group participation, would be directly associated with less depression and less suicidal thoughts and behaviour. However, only perceived support availability appears to have a general mental health effect (Thoits, 2011) in this sample, in that it is directly associated with less depression among ALHIV whether or not these adolescents are experiencing stigma. This is not the case for support group participation, which is not significantly associated with depression. Moreover, neither perceived social support availability nor group participation are directly associated with suicidal thoughts and behaviour in multivariate analyses.

\subsection{Stress-buffering effects of support resources}

Lastly, we hypothesised that these support resources would buffer the effects of stigma on depression and suicidal thoughts and behaviour. Findings do indeed point to stress-buffering protective effects of social support (Alloway and Bebbington, 1987) on the psychological outcomes of these HIV-positive adolescents. Both support resources investigated in these analyses appear to be moderating the negative effects of stigma on suicidal thoughts and behaviour. In particular, the combination of higher perceived social support and belonging to a support group appears to be particularly important to buffer the indirect effect of stigma, mediated by depression: it would in fact seem that (only) in the presence of both support resources, the mediated relationship between stigma and suicidal tendencies is not significant. Moreover, these individual support resources seem to be operating slightly differently to produce this stress-buffering effect: both perceived social support and support group participation are moderating the relationship between stigma and depression, but only perceived social support is moderating the effects of depression on suicidal thoughts and behaviour. Instead, with regard to direct effects of stigma, support group participation is what appears to make the difference: stigma did not have a significant effect on suicidal thoughts and behaviour for adolescents who attended a support group, regardless of their level of perceived social support. It is unclear how the observed direct effect of stigma in this model is operating, and may indicate the presence of other mediating mechanisms, for example psychological variables such as self-efficacy, selfesteem and hope (Casale et al., 2013; Thoits, 2011). Future research should explore these potential mechanisms, as well as possible moderating effects of support interventions on these pathways.

\subsection{Limitations}

This study has a number of limitations. It is subject to the risks of reporting bias and inaccuracies linked to self-reported data - although there are no known alternatives to reporting for factors such as experienced stigma and suicidal thoughts and behaviour. In addition, the data is cross-sectional and, despite theoretical assumptions around causality 
guiding these analyses, in some cases associations could be bi-directional: for example, poor mental health may negatively affect perceived stigma, prior suicidal attempts may increase stigma, and, in certain circumstances, participation or non-participation in a support group could potentially lead to stigma. In this regard future analyses with longitudinal data, and mixed methods research, could provide further useful insights. Also, given the complexity of these analyses and the focus on social resources, we chose to use an aggregate stigma variable rather than disaggregate by its individual sub-constructs. However, recent literature has highlighted the potential usefulness of exploring core psychosocial HIVstigma mechanisms - namely internalized stigma, enacted stigma and anticipated stigma distinctly (Earnshaw and Chaudoir, 2009; Pantelic et al., 2017), to obtain a more nuanced understanding of how HIV-related stigmas may be affecting health-seeking behaviours (Earnshaw et al., 2013; Turan et al., 2017). It may be useful for future research to unpack the relationship between different stigma mechanisms and mental health outcomes for ALHIV.

\subsection{Implications for future research and interventions}

Despite the above-indicated limitations, this paper contributes to a key gap in the literature. These are the first known analyses to explore the moderating effects of social support resources on the direct and indirect relationship between stigma and suicidal ideation and attempts, among ALHIV in a low-income setting. Findings reinforce the potential of social support as an important protective general mental health and stress-buffering resource for ALHIV. They also suggest that the distinct roles of specific support resources - representing different sources and/or types of support - are important to consider in understanding these overall effects. Perceived support availability and support group participation in this sample appear to be conceptually distinct in terms of their functional dimension (House and Khan, 1985) and support providers. It is likely that the types of instrumental and emotional support measured by the perceived support construct in this study (e.g. support with health care, practical help or general problem-solving) are being provided by individuals within the adolescent's informal family and community network, and not by support group facilitators or members. Clinic-based HIV support groups are instead likely to be providing predominantly informational and emotional support related to HIV treatment and the adolescent's experience of living with HIV, distinct from the support deriving from other naturally occurring relationships. Further enquiry would however be needed to determine the content and functioning of clinic support groups versus adolescents' other sources of support. It would also be useful for future research to help further our understanding of the effects and pathways through which these different support types and mechanisms may be operating simultaneously (House et al., 1988) - and possibly synergistically - to improve health behaviours and outcomes in this specific adolescent population.

Our findings also have several implications for interventions. First, they suggest that it is important to intervene as early as possible to strengthen support resources among ALHIV who are experiencing stigma and poor mental health, in order to mitigate further risks of deteriorating mental health and suicidal attempts. Support groups show potential as a component of effective mental health and suicide prevention interventions; they are 
also relatively low-cost initiatives with potentially high acceptability and sustainability in the medium-long term, since they draw from existing African community models (Verhoef, 2001). Moreover, while this paper focuses on depression and suicide, support groups may have several further beneficial effects in other domains, for example on social functioning, coping skills, psychological resilience, health service uptake and treatment adherence (Wouters et al., 2009; Wu and Li, 2013). These should be considered when assessing the potential costs and benefits of scaling up similar initiatives with public health resources.

It is noteworthy, however, that, despite potential benefits of support group participation, less than 20\% of ALHIV in this sample reported attending any form of clinic support group. Reasons may include a lack of support group availability at certain health facilities, time constraints, distance and travel cost to the meeting location, concerns linked to privacy or disclosure, or groups poorly aligned to needs specific to ALHIV (Adejumo et al., 2015). It would be useful to gain a better understanding of why participation in these groups is so low among ALHIV, and how to increase uptake and effectiveness through content and modalities that are desirable to adolescents. It is key that adolescents be involved in the development of these initiatives, since effective adult support interventions may not necessarily work for adolescents (Grimsrud et al., 2016).

Findings also suggest that attempting to prevent suicidal thoughts and behaviour through social support with ALHIV already experiencing depression may be more difficult, and likely require a greater focus on interventions that strengthen existing family and community support networks. These types of initiatives may also have the additional benefit of improving mental health more broadly through 'general health' protective effects (Thoits, 2011). However, structural interventions aiming to alter individuals' existing social networks and environments have shown mixed results and are often costly (Feaster et al., 2010), suggesting that we need to find alternative and preferably community-based solutions in settings with scarce (human and other) resources for health.

Lastly, results of these analyses show that our ability to protect ALHIV from negative psychosocial effects of HIV-related stressors is likely to be greater in the presence of multiple support resources. Adolescents in this sample that both perceived high social support and participated in support groups appeared to be more resilient than their peers. This suggests that there is value in working to strengthen multiple support resources available to ALHIV. While further research would be needed to determine long-term effects, our findings present an example of a support intervention that can be protective for adolescent psychosocial health, in a way that may be both distinct and complementary to the support deriving from naturally occurring relationships (Holt-Lanstad et al., 2010). These findings are noteworthy, given the challenges often faced by interventionists attempting to replicate positive effects of support deriving from 'informal' social relationships (Ertel et al., 2010; Holt-Lanstad et al., 2010). They point to the potential value of clinic-community collaborations and are particularly pertinent for low resource settings where public specialised psychiatric services are unfortunately scarce or nonexistent. 
Adolescents living with HIV in low and middle income countries should be a priority population group for the prevention of mental health disorders and suicide. Even where poor mental health and suicidal ideation do not result in suicidal attempts, they can lead to poor quality of life, high risk behaviours and lower retention in HIV treatment and care, resulting in higher HIV transmission and AIDS-related mortality (Lowenthal et al., 2014; Mutumba et al., 2015). These challenges are likely to intensify over the next decade, especially in the sub-Saharan African region, where adolescent populations and specifically adolescents living with HIV and dependent on life-long care and treatment continue to grow (Lowenthal et al., 2014). Better understanding risk and protective factors for psychosocial health, and developing more effective interventions to protect these adolescents' health, will be critical to their survival and realisation of full life potential.

\section{Authors' contributions}

As first author, $\mathrm{MC}$ took the lead in conceptualizing and writing the paper and conducted the statistical analysis with the support of MB. LC and ET had key roles in designing the research study and managing the research data. MP and MB contributed to the research design and to the choice of measurement tools. LC, ET, MC and MP were involved in the management of the field research. All authors contributed to and have approved the final manuscript.

\section{Funding}

The study was supported by the Nuffield Foundation under grant CPF/41513, but the views expressed are those of the authors and not necessarily those of the Foundation, the Regional Inter-Agency Task Team for Children Affected by AIDS - Eastern and Southern Africa (RIATT-ESA), the International AIDS Society through the CIPHER grant (155-Hod), Janssen Pharmaceutica N.V., part of the Janssen Pharmaceutical Companies of Johnson \& Johnson, the Evidence for HIV Prevention in Southern Africa (EHPSA), a UK aid programme managed by Mott MacDonald, Claude Leon Foundation [Fo8 559/C], Oak Foundation [R46194/AAoo1], UNFPA South Africa, UNICEF Eastern and Southern Africa, the John Fell Fund [103/757 and 161/033] and the Oxford University Clarendon-Green Templeton College Scholarship (ET, MP). Additional support for LC was provided by the European Research Council (ERC) under the European Union's Seventh Framework Programme [FP7/2007-2013]/ERC grant agreement no. 313421, the Philip Leverhulme Trust [PLP2014-095] and the project received the University of Oxford's ESRC Impact Acceleration Account [IAA-MT13-003, K1311-KEA-004 and 1602-KEA-189]. Additional suport for ET was provided from Elma Philanthropies. MB was supported by a New Independent Researcher Infrastructure Support Award from the Department of Health, Government of

Western Australia. The funding sources did not have any involvement in the writing of this paper or in the decision to submit the article for publication.

\section{Competing interests}

The authors understand and comply with the Journal's Conflict of Interest Policy. All the authors declare that they have no conflicts of interest. 


\section{Acknowledgements}

We are very grateful to the adolescents living with HIV and their caregivers who have generously shared their time and experiences for the purposes of this research. Much of the fight against HIV and AIDS relies upon people living with HIV continuing to put themselves forward and this research and our fight against HIV and AIDS is indebted to those past and present.

\section{Supplementary materials}

Supplementary material associated with this article can be found, in the online version, at doi:10.1016/j.jad.2018.10.102. 


\section{References}

Adejumo, O., Malee, K., Ryscavage, P., Hunter, S., Taiwo, B., 2015. Contemporary issues on the epidemiology and antiretroviral adherence of HIV-infected adolescents in sub-Saharan Africa: a narrative review. J. Int. AIDS Soc. 18 (20049). https://doi.org/10. 7448/IAS.18.1.20049. eCollection 2015.

Aiken, L., West, S., 1991. Multiple Regression: Testing and Interpreting Interactions. Sage, Thousand Oaks, CA.

Alloway, R., Bebbington, P., 1987. The buffer theory of social support - a review of the literature. Psychol. Med. 17, 91-108.

Bitew, H., Andargie, G., Tadesse, A., Belete, A., Fekadu, W., Mekonen, T., 2016. Suicidal ideation, attempt, and determining factors among HIV/AIDS patients, Ethiopia. Depress. Res. Treat. 2016, 1-6. https://doi.org/10.1155/2016/8913160.

Boyes, M., Cluver, L., 2013. Relationships among HIV/AIDS orphanhood, stigma, and symptoms of anxiety and depression in South African youth: a longitudinal investigation using a path analysis framework. Clin. Psychol. Sci. 1, 323-330.

Brandt, R., 2009. The mental health of people living with HIV/AIDS in Africa: a systematic review. Afr. J. AIDS Res. 8, 123-133.

Breet, E., Kagee, A., Seedat, S., 2014. HIV-related stigma and symptoms of posttraumatic stress disorder and depression in HIV-infected individuals: does social support play a mediating or moderating role? AIDS Care 26, 947-951.

Casale, M., Cluver, L., Crankshaw, T., Kuo, C., Lachman, J., Wild, L., 2015a. Direct and indirect effects of caregiver social support on adolescent psychological outcomes in two South African AIDS-affected communities. Am. J. Community Psychol. 55, 336-346.

Casale, M., Wild, L., Kuo, C., 2013. "They give us hope": HIV-positive caregivers' perspectives on the role of social support for health. AIDS Care 25, 1203-1209.

Casale, M., Wild, L., Cluver, L., Kuo, C., 2015b. Social support as a protective factor for depression among women caring for children in HIV-endemic South Africa. J Behav Med. 38, 17-27.

Catalan, J., Harding, R., Sibley, E., Clucas, C., Croome, N., Sherr, L., 2011. HIV infection and mental health: suicidal behaviour - systematic review. Psychol. Health Med. 16. Cluver, L., Orkin, M., Boyes, M., Sherr, L., 2015. Child and adolescent suicide attempts, suicidal behavior, and adverse childhood experiences in South Africa: a prospective study. J. Adolesc. Health 57, 52-59.

Cluver, L., Toska, E., Orkin, M., Meinck, F., Hodes, R., Yakubovich, A., Sherr, L., 2016. Achieving equity in HIV-treatment outcomes: can social protection improve adolescent ART-adherence in South Africa? AIDS Care 28, 73-82.

Cluver, L.D., Orkin, M., 2009. Cumulative risk and AIDS-orphanhood: Interactions of stigma, bullying and poverty on child mental health in South Africa. Soc. Sci. Med. 69, 1186-1193.

Cohen, S., Wills, T., 1985. Stress, social support and the buffering hypothesis. Psychol. Bull. 98, 310-357.

Dawson, J., 2018. Interpreting interaction effects. [WWW Document]. http://www. jeremydawson.co.uk/slopes.htm (accessed 10.2.17). 
Dow, D., Turner, E., Shayo, A., Mmbaga, B., Cunningham, C., O'Donnell, K., 2016. Evaluating mental health difficulties and associated outcomes among HIVpositive adolescents in Tanzania. AIDS Care 28, 825-833.

Earnshaw, V., Chaudoir, S., 2009. From conceptualizing to measuring HIV stigma: a review of HIV stigma mechanism measures. AIDS Behav. 13, 1160-1177.

Earnshaw, V., Smith, L., Chaudoir, S., Amico, R., Copenhaver, M., 2013. HIV stigma mechanisms and well-being among PLWH: a test of the HIV stigma framework. AIDS Behav. 17, 1785-1795.

Ertel, K., Glymour, M., Berkman, L., 2010. Social networks and health: a life course perspective integrating observational and experimental evidence. J. Soc. Pers. Relat. 26, 73-92.

Feaster, D., Brincks, A.M., Mitrani, V.B., Prado, G., Schwartz, S.J., Szapocznik, J., 2010. The efficacy of structural ecosystems therapy for HIV medication adherence with African American women. J. Fam. Psychol. 24, 51-59.

Gebremariam, E., Reta, M., Nasir, Z., Amdie, F., 2017. Prevalence and associated factors of suicidal ideation and attempt among people living with HIV/AIDS at Zewditu Memorial Hospital, Addis Ababa, Ethiopia: a cross-sectional study. Psychiatry J. 2017. https://doi.org/10.1155/2017/2301524.

Gellert, P., Häusler, A., Suhr, R., Gholami, M., Rapp, M., Kuhlmey, A., Nordheim, J., 2018. Testing the stress-buffering hypothesis of social support in couples coping with early-stage dementia. PLoS One 13, e0189849.

Gentz, S., Calonge Romano, I., Martínez-Arias, R., Ruiz-Casares, M., 2017. Predictors of mental health problems in adolescents living with HIV in Namibia. Child Adolesc. Ment. Health 22, 179-185.

Grimsrud, A., Lesosky, M., Kalombo, C., Bekker, L., Myer, L., 2016. Implementation and operational research: community-based adherence clubs for the management of stable antiretroviral therapy patients in Cape Town, South Africa: a cohort study. J. Acquir. Immune Defic. Syndr. 71, e16-e23.

Hayes, A., 2013. Introduction to Mediation, Moderation, and Conditional Process Analysis: A Regression-Based Approach. The Guilford Press, New York.

HIV Leadership through Accountability Programme: GNP and NAPWA SA, 2012. The People Living with HIV Stigma Index or Tambo Region Assessment. HIV Leadership through Accountability Programme: GNP and NAPWA SA, Germiston, South Africa.

Holt-Lanstad, J., Smith, T., Layton, B., 2010. Social relationships and mortality: a meta-analysis. PLoS Med. 7, e100031.

House, J., Khan, R., 1985. Measures and concepts of social support. In: Cohen, S., Syme, S. (Eds.), Social Support and Health. Academic Press, Orlando.

House, J., Umberson, D., Landis, K., 1988. Structures and processes of social support. Annu. Rev. Sociol. 14, 293-318.

Katz, T., Ryu, A.E., Onuegbu, A.G., Psaros, C., Weiser, S.D., Bangsberg, D.R., Tsai, A.C., 2013. Impact of HIV-related stigma on treatment adherence: systematic review and meta-synthesis. J. Int. AIDS Soc. 16. https://doi.org/10.7448/IAS.16.3.18640. 
Kessler, R., Avenevoli, S., Costello, E., Georgiades, K., Green, J., Gruber, M., He, J., Koretz, D., McLaughlin, K., Petukhova, M., Sampson, N., Zaslavsky, A., Merikangas, K., 2012. Prevalence, persistence, and sociodemographic correlates of DSM-IV disorders in the National Comorbidity Survey Replication Adolescent Supplement. Arch. Gen. Psychiatry 69, 372-380.

Kovacs, M., 1992. Children's Depression Inventory. Multi-Health Systems, Niagara Falls, NY.

Lecrubier, Y., Sheehan, D.V, Weiller, E., Amorim, P., Bonora, I., Harnett Sheehan, K., Dunbar, G.C., 1997. The Mini International Neuropsychiatric Interview (MINI). A short diagnostic structured interview: reliability and validity according to the CIDI. Eur. Psychiatry 12, 224-231.

Lewis, J., Abramowitz, S., J. Koenig, L., Chandwani, S., Orban, L., 2015. Negative life events and depression in adolescents with HIV: a stress and coping analysis. AIDS Care 27, 1265-1274.

Lowenthal, E., Bakeera-Kitaka, S., Marukutira, T., Chapman, J., Goldrath, K., Ferrand, R., 2014. Perinatally acquired HIV infection in adolescents from sub-Saharan Africa: a review of emerging challenges. Lancet Infect. Dis. 14, 627-639.

McDowell, T., Serovich, J., 2007. The effect of perceived and actual social support on the mental health of HIV-positive persons. AIDS Care 19, 1223-1229.

McKinnon, B., Gariépy, G., Sentenac, M., Elgar, F., 2016. Adolescent suicidal behaviours in 32 low- and middle-income countries. Bull. World Health Organ. 94, 340-350.

Mellins, C., Malee, K., 2013. Understanding the mental health of youth living with perinatal HIV infection: lessons learned and current challenges. J. Int. AIDS Soc. 16, 18593 .

Mutumba, M., Resnicow, K., Bauermeister, J., Harper, G., Musiime, V., Snow, R., Lepkowski, J., 2015. Development of a psychosocial distress measure for Ugandan adolescents living with HIV. AIDS Behav. 19, 380-392.

Page, R., Saumweber, J., Hall, P., Crookston, B., West, J., 2013. Multi-country, crossnational comparison of youth suicide ideation: findings from global school-based Health Surveys. Sch. Psychol. Int. 34, 540-555.

Pantelic, M., Boyes, M., Cluver, L., Meinck, F., 2017. HIV, violence, blame and shame: pathways of risk to internalized HIV stigma among South African adolescents living with HIV. J. Int. AIDS Soc. 20, 21771.

Pantelic, M., Boyes, M., Cluver, L., Thabeng, M., 2018. “They say HIV is a punishment from god or from ancestors": cross-cultural adaptation and psychometric assessment of an HIV stigma scale for South African adolescents living with HIV (ALHIV-SS). Child Indic. Res. 11, 207-223.

Patton, G., Coffey, C., Sawyer, S., Viner, R., Haller, D., Bose, K., Vos, T., 2009. Global patterns of mortality in young people: a systematic analysis of population health data. Lancet 374, 881-892.

Pillay, U., Roberts, B., Rule, S., 2007. South African Social Attitudes: Changing Times, Diverse Voices. Human Sciences Research Council, Pretoria, South Africa. 
Preacher, K., Hayes, A., 2008. Asymptotic and resampling strategies for assessing and comparing indirect effects in multiple mediator models. Behav. Res. Methods 40, 879-891.

RHRU, 2005. Lovelife: The National Survey of HIV and Sexual Behaviour among Young South Africans. RHRU, Johannesburg, South Africa.

Robbins, M., Szapocznik, J., Tejeda, M., Samuels, D., Ironson, G., Antoni, M., 2003. The protective role of the family and social support network in a sample of HIV-positive African American women: results of a pilot study. J. Black Psychol. 29, 17-37.

Rueda, S., Gibson, K., Rourke, S., Bekele, T., Gardner, S., Cairney, J., Team, T.O.C.S., 2012. Mastery moderates the negative effect of stigma on depressive symptoms in people living with HIV. AIDS Behav. 16, 690-699.

Rueda, S., Mitra, S., Chen, S., Gogolishvili, D., Globerman, J., Chambers, L., Wilson, M., Logie, C.H., Shi, Q., Morassaei, S., Rourke, S.B., 2016. Examining the associations between HIV-related stigma and health outcomes in people living with HIV/AIDS: a series of meta-analyses. BMJ 6, e011453.

Rukundo, G., Mishara, B., Kinyanda, E., 2016. Burden of suicidal ideation and attempt among persons living with HIV and AIDS in semiurban Uganda. AIDS Res. Treat. 2016, 1-9. https://doi.org/10.1155/2016/3015468.

Ryan, A., Willits, F., 2007. Family ties, physical health, and psychological well-being. J. Aging Health 19, 907-920.

Rzeszutek, M., 2017. Social support and posttraumatic growth in a longitudinal study of people living with HIV: the mediating role of positive affect. Eur. J. Psychotraumatol. 8, 1412225 .

Scharko, A., 2006. DSM psychiatric disorders in the context of pediatric HIV/AIDS. AIDS Care 18, 441-445.

Sheehan, D., Shytle, D., Milo, K., 2004. MINI KID: mini international neuropsychiatric interview for children and adolescents. English Version 4.o.

Sherbourne, C., Stewart, A., 1991. The MOS Social Support Survey. Soc. Sci. Med. 32, 705-714.

Shilubane, H., Ruiter, R., Bos, A., den Borne, B., James, S., Reddy, P., 2014. Psychosocial correlates of suicidal ideation in rural South African adolescents. Child Psychiatry Hum. Dev. 45, 153-162.

Slogrove, A., Mahy, M., Armstrong, A., Davies, M., 2017. Living and dying to be counted: What we know about the epidemiology of the global adolescent HIV epidemic. J. Int. AIDS Soc. 20, 21520.

Smith Fawzi, M.C., Eustache, E., Oswald, C., Louis, E., Surkan, P., Scanlan, F., Hook, S., Mancuso, A., Mukherjee, J., 2012. Psychosocial support intervention for HIVaffected families in Haiti: implications for programs and policies for orphans and vulnerable children. Soc. Sci. Med. 74, 1494-1503.

Suliman, S., 2002. Assessing Post-Traumatic Responses among South African Adolescents: A Comparison of Different Methods. University of Cape Town, Cape Town, South Africa. 
Teismann, T., Forkmann, T., Glaesmer, H., Egeri, L., Margraf, J., 2016. Remission of suicidal thoughts: findings from a longitudinal epidemiological study. J. Affect. Disord. 15, 723-725.

The South African Depression and Anxiety Group, 2016. Teen Suicide Infographic 2016. [WWW

Document].

http://www.sadag.org/index.php?option=com_content\&view= article\&id=2941:teen-suicide-infographic-2016\&catid=92\&Itemid=154 (accessed 2.14.18).

Thoits, P., 2011. Mechanisms linking soci health. J. Health Soc. Behav. 52, 145

Turan, B., Hatcher, A., Weiser, S., O. Johnson, M., Rice, W., Turan, J., 2017. Framing mechanisms linking HIV-related stigma, adherence to treatment, and health outcomes. Am. J. Public Health 6. https://doi.org/10.2105/AJPH.2017.303744.

Umberson, D., Montez, J., 2010. Social relationships and health: a flashpoint for health policy. J. Health Soc. Behav. 51, S54-S66.

UNFPA, 2014. The power of 1.8 billion adolescents, youth and the transformation of the future. https://www.unfpa.org/sites/default/files/pub-pdf/EN-SWOP14-Report_ FINAL-web.pdf (accessed 2.2.18).

Verhoef, G., 2001. Savings and survival in a modern African economy: informal savings organisations and poor people in South Africa. Historia Santiago 46, 519-542.

Wight, R., Aneshensel, C., LeBlanc, A., 2003. Stress buffering effects of family support in AIDS caregiving. AIDS Care 15, 595-613.

Wild, L., Flisher, A., Lombard, C., 2004. Suicidal ideation and attempts in adolescents: associations with depression and six domains of self-esteem. J. Adolesc. 27, 611-624.

Willis, N., Mavhu, W., Wogrin, C., Mutsinze, A., Kagee, A., 2018. Understanding the experience and manifestation of depression in adolescents living with HIV in Harare, Zimbabwe. PLoS One 13, e0190423. https://doi.org/10.1371/journal.pone. 0190423.

Wouters, E., Van Dammeb, W., Van Loona, F., van Rensburg, D., Meulemansa, H., 2009. Public-sector ART in the Free State Province, South Africa: community support as an important determinant of outcome. Soc. Sci. Med. 69, 1177-1185.

Wright, G., 2008. Findings from the indicators of poverty and social exclusion project: a profile of poverty using the socially perceived necessities approach. Key Report 7 .

Wright, K., Naar-King, S., Lam, P., Templin, T., Frey, M., 2007. Stigma scale revised: reliability and validity of a brief measure of stigma for HIV+ youth. J. Adolesc. Health 40, 96-98.

Wu, L., Li, X., 2013. Community-based HIV/AIDS interventions to promote psychosocial wellbeing among p le living with HIV/AIDS: a literature review. Health Psychol. Behav. Med. 1, 31 